\title{
Comparison of the Yield of Very Low Nicotine Content Cigarettes to the Top 100 United States Brand Styles*
}

\author{
by \\ Ed Carmines ${ }^{1}$ and I. Gene Gillman ${ }^{2}$ \\ ${ }^{I}$ Carmines Consulting, LLC, Scottsdale, AZ, USA \\ ${ }^{2}$ Enthalpy Analytical LLC, Durham, NC, USA
}

\section{SUMMARY}

The objective of this work was to compare the nicotine content and yield of new very low nicotine content cigarettes $\left(\mathrm{VLN}^{\mathrm{TM}}\right)$ to the top 100 cigarette brand styles in the United States. Nicotine in tobacco filler and nicotine in smoke were measured. On a dry weight basis, VLNTM cigarettes averaged $0.5 \mathrm{mg}$ of nicotine/g tobacco as compared to 19.4 for the top 100 brand styles in the United States. On a cigarette basis, VLN ${ }^{\mathrm{TM}}$ cigarettes contained $0.27 \mathrm{mg}$ of nicotine compared to $12 \mathrm{mg}$ for the top brands. On an ISO smoke yield basis, VLNTM produced $0.03 \mathrm{mg}$ nicotine/cig compared to $0.903 \mathrm{mg}$ nicotine/cig for the leading brands. VLNTM cigarettes contained $>95 \%$ less nicotine on a per gram basis of tobacco or per cigarette basis than the top 100 brand styles in the U.S. VLNTM cigarettes yielded $>95 \%$ less nicotine in smoke than the top brand styles in the United States. [Beitr. Tabakforsch. Int. 28 (2019) 253-266]

\section{KEYWORDS}

Tobacco; cigarettes; nicotine, SPECTRUM ${ }^{\circledR}, \mathrm{VLN}, \mathrm{VLNC}$, very low nicotine

\section{ZUSAMMENFASSUNG}

Ziel der vorliegenden Arbeit war ein Vergleich von Nikotingehalt und Nikotinausbeute einer neuen Zigarette mit sehr geringem Nikotingehalt (VLN ${ }^{\mathrm{TM}}$ ) mit den 100 führenden Zigarettenprodukten in den USA. Es wurde das Nikotin im Füllstoff und das Nikotin im Rauch gemessen.

\begin{abstract}
Als Trockengewicht ergab sich für die VLNTM-Zigaretten durchschnittlich $0,5 \mathrm{mg}$ Nikotin/g Tabak im Vergleich zu 19,4 bei den 100 führenden Produkten in den USA. Pro Zigarette enthielten die VLN ${ }^{\mathrm{TM}}$-Zigaretten $0,27 \mathrm{mg}$ Nikotin im Vergleich zu $12 \mathrm{mg}$ bei den wichtigsten Produkten. Hinsichtlich der ISO-Rauchausbeute gaben die VLNTM Zigaretten $0,03 \mathrm{mg}$ Nikotin/Zigarette ab verglichen mit $0,903 \mathrm{mg}$ der führenden Produkte. VLNTM - Zigaretten enthielten $>95 \%$ weniger Nikotin pro Gramm Tabak und pro Zigarette als die 100 führenden Zigarettenprodukte in den USA. Für VLNTM-Zigaretten wurde zudem eine im Vergleich zu den führenden US-Produkten um $>95 \%$ geringere Nikotinausbeute im Rauch ermittelt. [Beitr. Tabakforsch. Int. 28 (2019) 253-266]
\end{abstract}

\section{RESUME}

L'objectif de la présente étude était un comparatif de la teneur et du rendement en nicotine de nouvelles cigarettes à très faible teneur en nicotine $\left(\mathrm{VLN}^{\mathrm{TM}}\right)$ par rapport aux styles de cigarettes répertoriés dans le top 100 des marques commercialisées aux Etats-Unis. La nicotine fut mesurée dans la matière de remplissage et dans la fumée. Les cigarettes VLN ${ }^{\mathrm{TM}}$ affichèrent une moyenne de $0,5 \mathrm{mg}$ de nicotine par gramme de tabac, exprimée en fonction du poids sec, contre 19,4 mg dans le cas des styles de cigarettes du top 100 des marques commercialisées aux EtatsUnis. Considérant l'ensemble de la cigarette, les cigarettes VLN $^{\mathrm{TM}}$ contenaient $0,27 \mathrm{mg}$ de nicotine par cigarette, contre $12 \mathrm{mg}$ pour les marques du classement. Selon la méthode ISO de détermination du rendement de la fumée, les cigarettes VLNTM produisaient $0,03 \mathrm{mg}$ de nicotine par cigarette, contre $0,903 \mathrm{mg}$ de nicotine par cigarette dans le

*Received: $14^{\text {th }}$ April 2019 - accepted: $19^{\text {th }}$ July 2019 
cas des grandes marques. Les cigarettes VLNTM contenaient plus de $95 \%$ de nicotine en moins par gramme de tabac et par cigarette que les styles de cigarettes répertoriés dans le top 100 des marques commercialisées aux Etats Unis. Les cigarettes VLNTM libéraient plus de 95\% de nicotine en moins dans la fumée que les produits des grandes marques commercialisées aux USA. [Beitr. Tabakforsch. Int. 28 (2019) 253-266]

\section{INTRODUCTION}

It is well known that nicotine is the addictive agent in tobacco. In 1994, Drs. BENOWITZ and HENNINGFIELD proposed reducing nicotine levels of all cigarettes over a 10-15-year period (1). Over the intervening years, literally over a hundred scientific studies were published, evaluating the concept and benefits of very low nicotine cigarettes. In a June 16, 2010 press release, Dr. David Kessler, at the time FDA Commissioner, recommended "The FDA should quickly move to reduce nicotine levels in cigarettes to nonaddictive levels"(2). Shortly thereafter in a Washington Post newspaper article, Dr. Kessler said that the amount of nicotine in a cigarette should drop from about $10 \mathrm{mg}$ to less than $1 \mathrm{mg}$ (3). In 2017, Dr. Scott Gottlieb, at the time FDA Commissioner, announced a new more comprehensive approach to effective tobacco regulation based on reducing the nicotine levels in cigarettes so that they are minimally or non-addictive (4). As part of the new plan, on March 16, 2018, FDA published an advance notice of proposed rulemaking (ANPRM) announcing an intention to issue a proposed rule that, when finalized and effective, would limit the amount of nicotine in all cigarettes sold in the United States so that they are minimally addictive or nonaddictive (5). There are no commercially available cigarettes that meet the proposed standard of $0.2-0.7 \mathrm{mg}$ of nicotine per cigarette.

In 2011, 22nd Century developed the SPECTRUM ${ }^{\circledR}$ line of research cigarettes in collaboration with the National Institute on Drug Abuse (NIDA), the FDA, the National Cancer Institute and the Centers for Disease Control and Prevention. These cigarettes are made available to researchers through NIDA's Drug Supply Program. The SPECTRUM ${ }^{\circledR}$ product line consists of a series of cigarette styles that vary in nicotine content - from very low $(\sim 0.4 \mathrm{mg} / \mathrm{g}$ tobacco) to relatively high nicotine contents and yields. The nicotine smoke yields range from 0.02 to $1.2 \mathrm{mg}$ nicotine/cig and from 3 to $16 \mathrm{mg}$ of "tar"/cig (6). SPECTRUM ${ }^{\circledR}$ products are available in 24 styles, in both regular and menthol versions, with 8 levels of nicotine in their tobacco. The amount of nicotine in the various styles of SPECTRUM ${ }^{\circledR}$ cigarettes is controlled by blending conventional nicotine content tobacco and tobacco that has been genetically modified $^{1}$ and selected to have low levels of nicotine. The lowest nicotine level SPECTRUM® ciga-

1 The tobacco used in SPECTRUM $®$ and VLNTM cigarettes is derived from a low alkaloid strain of Burley 21 tobacco. The Animal and Plant Health Inspection Service (APHIS) of the United States Department of Agriculture (USDA) prepared an environmental assessment of tobacco line Vector 21-41 and granted a petition to designate this product as non-regulated (33). rettes contain only very low nicotine tobacco. As nicotine production in tobacco is controlled by multiple genes in the plant, 22nd Century's technology involves blocking or downregulating several genes in the tobacco plant and then selecting for tobacco plant lines that produce the lowest levels of nicotine. SPECTRUM $®$ cigarettes are made with the Company's proprietary Burley tobacco (strain Vector 21-41) at a target nicotine specification level of $0.5 \mathrm{mg} / \mathrm{g}$ of tobacco filler (dry weight). The low nicotine level cigarettes are often stated to contain a nominal level of $0.4 \mathrm{mg}$ of nicotine/g of tobacco. These research cigarettes have been produced since 2011 with a target nicotine level of $0.5 \mathrm{mg}$ nicotine/g of dry tobacco filler weight basis accounting for variations in water content.

22nd Century's proprietary low nicotine tobacco was developed in 1998 and has since been used in many cigarettes under different names, including X-22, Xodus, Magic, MAGIC 0, MAGIC 2, Quest $\AA$, and the SPECTRUM ${ }^{\circledR}$ research cigarettes. Quest ${ }^{\circledR}$ cigarettes were developed and marketed by Vector Tobacco, Inc. under license from 22nd Century from 2002 to 2009. The family of Quest ${ }^{\circledR}$ products included Quest ${ }^{\circledR} 1$ (a cigarette made of conventional tobacco), Quest $\AA 2$ (a cigarette containing a blend of conventional tobacco and very low nicotine tobacco), and Quest $\AA 3$ (a cigarette made exclusively with very low nicotine tobacco). Quest cigarettes were marketed to reduce nicotine uptake and nicotine dependency. In 2006, Vector Tobacco filed an Investigational New Drug (IND) Application (number 69,185) with the U.S. FDA for the Quest products with very low nicotine tobacco. Stepdown reduction in nicotine levels in test cigarettes followed by nicotine replacement therapy (patches) did not affect abstinence at 3 and 6 months when compared to smoking the usual brand followed by nicotine replacement therapy (7). When Vector stopped the sales of Quest, 22nd Century developed X-22 cigarettes with the intent of obtaining a smoking cessation claim. In 2011, 22nd Century conducted a clinical study with X-22 under IND 103,589. The 4-week study failed to demonstrate cessation efficacy and the clinical development was paused.

22nd Century has developed a version of the SPECTRUM ${ }^{\circledR}$ research cigarette for commercial distribution. This product, trademarked VLN, is exactly the same as the lowest level of nicotine versions of SPECTRUM ${ }^{\circledR}$. The SPECTRUM ${ }^{\circledR}$ Nicotine Research Cigarettes (NRC) 102 and 103 will be commercially available (after FDA approval) as VLN ${ }^{\mathrm{TM}}$ Kings and VLN ${ }^{\mathrm{TM}}$ Menthol Kings, respectively. The goal of this research was to evaluate how the nicotine content and yield of VLNTM cigarettes compared to currently marketed cigarettes.

\section{METHODS}

\section{Cigarettes}

The top 100 cigarette brand styles in the U.S. were determined by purchasing sales data from Management Science Associates, Inc. (MSA). A one-time aggregate of 52 weeks of data for calendar year 2017, comprised of the week ending January 7, 2017, to the week ending December 30, 2017, was obtained. The data was U.S. nationwide sales 
data (in cartons) for warehouse-delivered products in the convenience store channel made up of both chain and independent stores. The United States convenience store channel universe is made up of $154,000+$ stores. MSA collects data from over 60,000 stores. From these 60,000 , a sub-set of approximately 30-32,000 convenience stores, both chain and independent, are used to project data to the size of the total convenience store channel. The raw sales data (in cartons) from MSA was categorized at the SKU (stock keeping unit; the bar code on the side of the pack) level. Identical products having different SKUs (because they were purchased with a coupon, or another form of discount, or if they have a slight difference in description) were combined. For testing purposes, the commercial cigarettes were purchased from retail outlets in the Richmond, VA metropolitan area during July 2018. VLN ${ }^{\mathrm{TM}}$ cigarettes were provided by 22 nd Century Group, Inc. The top 100 brand styles represented more than $80 \%$ of all cigarettes sold in the U.S. The brand styles tested are listed in Table 1. Some of the brand styles were discontinued. The next product on the list was tested.

VLNTM King and VLNTM Menthol King are the same cigarettes as the SPECTRUM ${ }^{\circledR}$ NRC102 and NRC103 research cigarettes produced by 22nd Century for NIDA. The only difference between VLNTM King and VLNTM Menthol King is that menthol has been added to the filter of the Menthol King product. All other tobacco, materials, and ingredients are the same in the products. Table 2 shows a comparison of the measured physical properties of $\mathrm{VLN}^{\mathrm{TM}}$ and SPECTRUM ${ }^{\circledR}$ cigarettes.

\section{Sample analysis}

Mainstream smoke collection and analysis of cigarettes were performed using an SM 450 20-port linear smoking machine (Cerulean, Milton Keyes, UK) under ISO conditions of $35 \mathrm{~mL}$ volume, $2 \mathrm{~s}$ duration with a $60 \mathrm{~s}$ puff period. Twenty replicates were analyzed for "tar", nicotine, and carbon monoxide yields. Analysis was conducted following the relevant ISO standards for the analysis of nicotine, water, $\mathrm{CO}$ and Nicotine Free Dry Particulate Matter (NFDPM) in mainstream cigarette smoke (8-12). The ISO method for the analysis of nicotine in mainstream smoke, ISO 10315, does not cover VLN ${ }^{\mathrm{TM}}$ tobacco products because of the low nicotine levels. The work by BROWN et al. has demonstrated that with the addition of lower calibration standards, the method is suitable for the analysis of nicotine in the mainstream smoke of VLN products (13). At the time of the analyses, all analytical methods were accredited to the ISO 17025 standard $(14,15)$. Cigarette filler analysis was conducted from tobacco removed from conditioned cigarettes. Tobacco was composited from 200 cigarettes per brand style. Before analysis, tobacco was ground to a uniform particle size. Nicotine content of the commercial brands was determined by gas chromatography/flame ionization detector (GC-FID) following the CDC method (16) while nicotine content of the VLN products was determined by gas chromatography/ mass spectrometry (GC-MS) following CORESTA Recommend method 87 (17). Tobacco water content was determined by following the three-hour CDC oven volatiles method (16).

\section{Determination of “tar", nicotine, and water}

Total particulate matter (TPM) from cigarette smoke was collected on a Cambridge filter pad and extracted in $20 \mathrm{~mL}$ of 2-propanol (MilliporeSigma, Burlington, MA, USA, OmniSolv grade, CAS \#67-63-0) containing $0.5 \mathrm{~mL} / \mathrm{L}$ of n-heptadecane (Alfa-Aesar, Ward Hill, MA, USA, 99\%, CAS \#629-78-7) and $5 \mathrm{~mL} / \mathrm{L}$ of ethanol (Sigma-Aldrich, St. Louis, MO, USA, 200 proof anhydrous, CAS \#64-17-5) as internal standards. An aliquot of the sample solution was analyzed with an Agilent (Santa Clara, CA, USA) 6890N gas chromatograph equipped with a flame ionization detector (FID) and a thermal conductivity detector. The nicotine was analyzed on the FID channel using a custom stainless steel Supelco (Bellefonte, PA, USA) column measuring $6 \mathrm{ft} \times 1 / 8$ in $\times 2.1 \mathrm{~mm}$ with $7 \%$ Carbowax, $3 \%$ OS-138, $2 \% \mathrm{KOH}, 80 / 100$ Chromosorb WHP packing. The water was analyzed on the TCD channel using a custom stainless steel Supelco column measuring $10 \mathrm{ft} \times 1 / 8$ in $\times$ $2.1 \mathrm{~mm}$ with 80/100 Porapak QS packing. The "tar" (nicotine free dry particulate matter - NFDPM) was calculated by subtracting resulting nicotine and water from the TPM. The limit of detection under ISO 3308 for nicotine and water was $1.2 \mu \mathrm{g} / \mathrm{cig}$ and $80 \mu \mathrm{g} / \mathrm{cig}$ respectively while the limit of detection under ISO 10315 for nicotine and water was $2.0 \mu \mathrm{g} / \mathrm{cig}$ and $133 \mu \mathrm{g} / \mathrm{cig}$ respectively.

\section{Determination of carbon monoxide by non-dispersive infrared absorption}

The vapor phase of the smoke was collected in gas sampling bags attached to the smoking machine. A Cerulean non-dispersive infrared absorption detector (NDIR) was used to measure the $\mathrm{CO}$ concentration in the vapor phase in percent by volume $(\%$ vol $)$. The NDIR detector was calibrated using specialty gases from ARC3 (Richmond, VA) with certified concentrations of $1 \%, 3 \%$, and $6 \%$ (vol) $\mathrm{CO}$ in nitrogen. Using the number of cigarettes, the cigarette puff count, the puff volume, and ambient temperature and humidity conditions, the \% $\mathrm{CO}$ was converted to $\mathrm{mg} / \mathrm{cig}$.

\section{Determination of nicotine in tobacco filler by GC-FID}

$5 \mathrm{~mL}$ of $2 \mathrm{~N}$ sodium hydroxide (Fluka, Morristown, $\mathrm{NJ}$, USA, CAS \#1310-73-2) was added to the tobacco $(1.00 \mathrm{~g} \pm 0.1 \mathrm{~g})$ and allowed to stand for $15 \mathrm{~min} .50 \mathrm{~mL}$ of methyl tert-butyl ether (Macron, Allentown, PA, CAS \#1634-04-4) containing $0.4 \mathrm{mg} / \mathrm{mL}$ of the internal standard quinoline (Alpha-Aesar, 98\%, CAS \#91-22-5) was then added and the sample was placed on an orbital shaker for 2 hours at 200 RPM. An aliquot of the organic layer was analyzed with an Agilent 7890 gas chromatograph equipped with a flame ionization detector. Analyte separation was achieved using an Agilent J\&W HP-5 $30 \mathrm{~m} \times$ $320 \mu \mathrm{m} \times 0.25 \mu \mathrm{m}$ column. The limit of detection for this method was approximately $150 \mu \mathrm{g} / \mathrm{g}$ tobacco. 
Table 1. Top 100 brands in U.S. by unit sales.

\begin{tabular}{|c|c|c|c|c|}
\hline Manufacturer & Brand family & Item description & Rank & Notes \\
\hline Altria Group & Marlboro & Marlboro Gold King & 1 & \\
\hline Altria Group & Marlboro & Marlboro Red King & 2 & \\
\hline Reynolds American, Inc & Newport & Newport Menthol Green 100 & 3 & \\
\hline Reynolds American, Inc & Newport & Newport Menthol Green King & 4 & \\
\hline Altria Group & Marlboro & Marlboro Special Blend Gold King & 5 & \\
\hline Altria Group & Marlboro & Marlboro Gold 100 & 6 & \\
\hline Reynolds American, Inc & Pall Mall & Pall Mall Red 100 & 7 & \\
\hline Reynolds American, Inc & Camel & Camel Blue King & 8 & \\
\hline Altria Group & Marlboro & Marlboro Red 100 & 9 & \\
\hline Altria Group & Marlboro & Marlboro Special Blend Gold 100 & 10 & \\
\hline Altria Group & Marlboro & Marlboro Silver King & 11 & \\
\hline Altria Group & Marlboro & Marlboro Special Blend Black 100 & 12 & \\
\hline Altria Group & Marlboro & Marlboro Special Blend Red 100 & 13 & \\
\hline Altria Group & Marlboro & Marlboro Special Blend Red King & 14 & \\
\hline Altria Group & Marlboro & Marlboro Special Blend Black King & 15 & \\
\hline Reynolds American, Inc & Pall Mall & Pall Mall Blue (Light) 100 & 16 & \\
\hline Altria Group & Marlboro & Marlboro Menthol Gold King & 17 & \\
\hline Reynolds American, Inc & Camel & Camel Crush King & 18 & \\
\hline Altria Group & Marlboro & Marlboro Green (Menthol) King & 19 & \\
\hline Altria Group & Marlboro & Marlboro 72 Red & 20 & \\
\hline Reynolds American, Inc & Camel & Camel Menthol Silver King & 21 & Disc. ${ }^{1}$ \\
\hline Altria Group & L\&M & L\&M Full Flavor King & 22 & \\
\hline Altria Group & L\&M & L\&M 100 (Full Flavor) & 23 & \\
\hline Reynolds American, Inc & Pall Mall & Pall Mall Blue (Light) King & 24 & \\
\hline Reynolds American, Inc & Pall Mall & Pall Mall Red King & 25 & \\
\hline Reynolds American, Inc & Newport & Newport Non-Menthol 100 & 26 & \\
\hline Reynolds American, Inc & Camel & Camel Menthol Green King & 27 & Disc. $^{2}$ \\
\hline Altria Group & Marlboro & Marlboro Silver 100 & 28 & \\
\hline Reynolds American, Inc & Camel & Camel Full Flavor Filter King & 29 & \\
\hline ITG Brands LIC & Kool & Kool Menthol King & 30 & \\
\hline Reynolds American, Inc & Pall Mall & Pall Mall Menthol 100 & 31 & \\
\hline Altria Group & Marlboro & Marlboro Blend 27 King & 32 & \\
\hline Altria Group & L\&M & L\&M 100 Menthol & 33 & \\
\hline Altria Group & Marlboro & Marlboro 72 Gold & 34 & \\
\hline Altria Group & L\&M & L\&M Blue King & 35 & \\
\hline ITG Brands Llc & Winston & Winston Red King & 36 & \\
\hline Altria Group & Marlboro & Marlboro Nxt King & 37 & \\
\hline Altria Group & Marlboro & Marlboro Menthol 100 & 38 & \\
\hline ITG Brands LIC & Maverick & Maverick Menthol 100 & 39 & \\
\hline Reynolds American, Inc & Camel & Camel 99 Full Flavor Bx & 40 & \\
\hline ITG Brands LIC & Winston & Winston Gold King & 41 & \\
\hline Santa Fe Tobacco & Natural Am Spirits & Natural American Spirit Yellow Mellow King & 42 & \\
\hline Altria Group & L\&M & L\&M Blue 100 & 43 & \\
\hline Altria Group & L\&M & L\&M Menthol King & 44 & \\
\hline Reynolds American, Inc & Pall Mall & Pall Mall Orange (Ultra Light) 100 & 45 & \\
\hline Reynolds American, Inc & Newport & Newport Non-menthol King & 46 & \\
\hline Altria Group & Marlboro & Marlboro Smooth Menthol King & 47 & \\
\hline Santa Fe Tobacco & Natural Am Spirits & Natural American Spirit Blue King & 48 & \\
\hline Altria Group & Marlboro & Marlboro Menthol Gold 100 & 49 & \\
\hline Altria Group & Marlboro & Marlboro Special Blend Black Menthol 100 & 50 & \\
\hline Altria Group & Parliament & Parliament White King & 51 & \\
\hline ITG Brands LIC & Kool & Kool Green 100 & 52 & \\
\hline Reynolds American, Inc & Camel & Camel 99 Blue & 53 & \\
\hline Altria Group & Marlboro & Marlboro Special Blend Black Menthol King & 54 & \\
\hline Reynolds American, Inc & Pall Mall & Pall Mall White Menthol 100 & 55 & \\
\hline Altria Group & Marlboro & Marlboro 72 Silver & 56 & \\
\hline ITG Brands Llc & Winston & Winston Gold 100 & 57 & \\
\hline Altria Group & Marlboro & Marlboro Red Label King & 58 & \\
\hline Altria Group & Marlboro & Marlboro 72 Menthol Green & 59 & \\
\hline Reynolds American, Inc & Camel & Camel Turkish Blend Royal Filter King & 60 & \\
\hline
\end{tabular}


Table 1. Continued

\begin{tabular}{|c|c|c|c|c|}
\hline Manufacturer & Brand family & Item description & Rank & Notes \\
\hline Reynolds American, Inc & Pall Mall & Pall Mall Menthol King & 61 & \\
\hline ITG Brands LIC & Maverick & Maverick Full Flavor 100 & 62 & \\
\hline Reynolds American, Inc & Pall Mall & Pall Mall Black Menthol 100 & 63 & \\
\hline Reynolds American, Inc & Camel & Camel Wides Red King & 64 & \\
\hline Liggett Vector Brands & Pyramid & Pyramid Red 100 & 65 & \\
\hline Liggett Vector Brands & Eagle 20's & Eagle 20's Red 100 & 66 & \\
\hline Reynolds American, Inc & Camel & Camel Crush Menthol Silver King & 67 & \\
\hline Altria Group & Marlboro & Marlboro Southern Cut King & 68 & \\
\hline ITG Brands Llc & Winston & Winston Red 100 & 69 & \\
\hline Reynolds American, Inc & Newport & Newport Menthol Gold King & 70 & \\
\hline Altria Group & Marlboro & Marlboro Silver Menthol King & 71 & \\
\hline Reynolds American, Inc & Pall Mall & Pall Mall Orange 85 & 72 & \\
\hline Reynolds American, Inc & Camel & Camel Crush Menthol King & 73 & \\
\hline Altria Group & Marlboro & Marlboro Edge King & 74 & \\
\hline ITG Brands LIC & Kool & Kool Blue (Mild) King & 75 & \\
\hline Altria Group & Marlboro & Marlboro Smooth Menthol 100 & 76 & \\
\hline Santa Fe Tobacco & Natural Am Spirits & Natural American Spirit Organic Turquoise King & 77 & \\
\hline ITG Brands LIC & USA Gold & USA Gold Red King & 78 & \\
\hline Altria Group & Marlboro & Marlboro Black Menthol 100 & 79 & \\
\hline Altria Group & Marlboro & Marlboro Red Label 100 & 80 & \\
\hline Altria Group & Marlboro & Marlboro Black Menthol King & 81 & \\
\hline ITG Brands LIc & Salem & Salem Menthol Gold King & 82 & \\
\hline Reynolds American, Inc & Misty & Misty 120 Menthol (Light Green) & 83 & \\
\hline Santa Fe Tobacco & Natural Am Spirits & Natural American Spirit Menthol Light Green King & 84 & \\
\hline Liggett Vector Brands & Pyramid & Pyramid Blue 100 & 85 & \\
\hline ITG Brands Llc & Salem & Salem Slim Menthol 100 & 86 & \\
\hline ITG Brands LIC & Maverick & Maverick Gold (Light) 100 & 87 & \\
\hline Altria Group & Marlboro & Marlboro Silver Menthol 100 & 88 & \\
\hline Reynolds American, Inc & Camel & Camel Turkish Blend Gold King & 89 & \\
\hline Altria Group & Marlboro & Marlboro 83 & 90 & \\
\hline Altria Group & Marlboro & Marlboro Midnight Menthol King & 91 & \\
\hline ITG Brands LIC & USA Gold & USA Gold (Light) King & 92 & \\
\hline Reynolds American, Inc & Misty & Misty 120 Blue & 93 & \\
\hline Altria Group & Marlboro & Marlboro 72 Menthol Blue & 94 & Disc. $^{3}$ \\
\hline Reynolds American, Inc & Newport & Newport Menthol Gold 100 & 95 & \\
\hline Santa Fe Tobacco & Natural Am Spirits & Natural American Spirit Organic Mellow Gold King & 96 & \\
\hline ITG Brands LIc & USA Gold & USA Gold Red 100 & 97 & \\
\hline Altria Group & L\&M & L\&M Bold Menthol 100 & 98 & \\
\hline Reynolds American, Inc & Camel & Camel Turkish Blend Jade Silver King & 99 & \\
\hline ITG Brands Llc & Salem & Salem Green King & 100 & \\
\hline ITG Brands LIc & Winston & Winston White King & 101 & \\
\hline Reynolds American, Inc & Newport & Newport Menthol Smooth Select 100 & 102 & \\
\hline Altria Group & Marlboro & Marlboro Menthol Blue King & 103 & \\
\hline
\end{tabular}

1 Disc. = Discontinued - cannot test. The non-crush versions of the varieties of Camel Menthol products have been phased out and are no longer available for purchase. The sales of Camel Menthol Silver King (non-crush) was ranked \#21 of 100 in 2017. The sales of Camel Crush Menthol Silver King were ranked \#67 of 100, so the crush version of Camel Menthol Silver King was evaluated. Since item \#21 on the list no longer exists in the market, the next item down on the list, ranked \#102, Newport Menthol Smooth Select 100 size was chosen.

2 Disc. = Discontinued - cannot test. The non-crush versions of the varieties of Camel Menthol products have been phased out and are no longer available for purchase. The sales of Camel Menthol Green King (non-crush) was ranked \#27 of 100 in 2017. The sales of Camel Crush Menthol King were ranked \#73 of 100, so the crush version of Camel Menthol Green King was evaluated. Since item \#27 on the list no longer exists in the market, the next item down on the list, ranked \#101, Winston White King size was chosen.

3 Disc. = Discontinued - cannot test. Marlboro 72 Menthol Blue was discontinued in 2017. It ranked \#94 of 100 for sales on the 2017 list of products. Since it is not available for purchase it was replaced it with \#103 on the list, Marlboro Menthol Blue King Size. 
Table 2. Comparison of the measured physical properties of SPECTRUM and VLN cigarettes.

\begin{tabular}{|c|c|c|c|c|c|}
\hline Parameter & & $\begin{array}{c}\text { SPECTRUM King } \\
\text { NRC102 }\end{array}$ & VLN $^{T M}$ King & $\begin{array}{c}\begin{array}{c}\text { SPECTRUM Menthol } \\
\text { King NRC103 }\end{array} \\
\end{array}$ & VLN ${ }^{\mathrm{TM}}$ Menthol King \\
\hline \multirow[t]{2}{*}{ Cigarette length $(\mathrm{mm})$} & Mean & 82.8 & 82.9 & 82.9 & 82.9 \\
\hline & SD & 0.1 & 0.1 & 0.1 & 0.1 \\
\hline \multirow[t]{2}{*}{ Tipping length (mm) } & Mean & 30.2 & 30.2 & 30.1 & 30.3 \\
\hline & SD & 0.4 & 0.3 & 0.4 & 0.3 \\
\hline \multirow[t]{2}{*}{ Filter length (mm) } & Mean & 25.0 & 25.1 & 25.0 & 25.2 \\
\hline & SD & 0.0 & 0.2 & 0.0 & 0.3 \\
\hline \multirow[t]{2}{*}{ Rod length (mm) } & Mean & 57.8 & 57.9 & 57.9 & 57.7 \\
\hline & SD & 0.1 & 0.1 & 0.1 & 0.3 \\
\hline \multirow[t]{2}{*}{ Permeability banded (CU) } & Mean & 13.6 & 13.5 & 15.0 & 14.5 \\
\hline & SD & 4.0 & 2.6 & 4.5 & 5.4 \\
\hline \multirow[t]{2}{*}{ Permeability non-banded (CU) } & Mean & 77.6 & 81.5 & 79.2 & 83.3 \\
\hline & SD & 6.4 & 4.7 & 12.5 & 10.4 \\
\hline \multirow[t]{2}{*}{ Cigarette weight (g) } & Mean & 0.900 & 0.936 & 0.963 & 0.932 \\
\hline & SD & 0.018 & 0.021 & 0.026 & 0.017 \\
\hline \multirow[t]{2}{*}{ Tobacco weight (mg) } & Mean & 650 & 687 & 647 & 650 \\
\hline & SD & 21 & 23 & 16 & 20 \\
\hline \multirow[t]{2}{*}{ Circumference (mm) } & Mean & 24.9 & 24.8 & 24.8 & 25.0 \\
\hline & SD & 0.1 & 0.1 & 0.1 & 0.1 \\
\hline \multirow[t]{2}{*}{ Diameter (mm) } & Mean & 7.90 & 7.89 & 7.91 & 7.95 \\
\hline & SD & 0.03 & 0.02 & 0.03 & 0.02 \\
\hline \multirow[t]{2}{*}{ Tip ventilation (\%) } & Mean & 14.1 & 12.5 & 14.8 & 14.0 \\
\hline & SD & 1.5 & 1.1 & 1.7 & 1.3 \\
\hline \multirow[t]{2}{*}{ Pressure drop "open" } & Mean & 121.9 & 119.0 & 119.0 & 113.0 \\
\hline & SD & 5 & 4 & 4 & 4 \\
\hline \multirow[t]{2}{*}{ Plug resistance to draw } & Mean & 98.7 & 91.6 & 88.8 & 92.2 \\
\hline & SD & 5.5 & 4.2 & 5.1 & 6.0 \\
\hline \multirow{2}{*}{ Filter weight (g) } & Mean & 0.200 & 0.222 & 0.220 & 0.220 \\
\hline & SD & 0.003 & 0.003 & 0.003 & 0.003 \\
\hline
\end{tabular}

\section{Determination of nicotine in tobacco filler by GC/MS}

$4 \mathrm{~mL}$ of $5 \mathrm{~N} \mathrm{NaOH}$ (Fisher Chemical, Fair Lawn, NJ, USA) was added to the tobacco $(0.5 \mathrm{~g} \pm 0.1 \mathrm{~g})$ into a $50 \mathrm{~mL}$ conical tube and allowed to stand for $30 \mathrm{~min} .40 \mathrm{~mL}$ of methanol (Fisher, distilled in glass) containing $50 \mu \mathrm{g} / \mathrm{mL}$ of the internal standard quinoline (Alfa-Aesar P/N 43225) was added to the conical tube along with 2 grinding stones (US Stoneware, East Palestine, OH, USA, P/N Brun050-090). The sample was then placed on the 2010 Geno-grinder (Spex, Metuchen, NJ, USA) for $30 \mathrm{~min}$ at 1000 RPM. Samples were removed from the grinder and placed into a centrifuge (Sorvall Legend XT by Thermo Fisher Scientific, Waltham, MA, USA) for $10 \mathrm{~min}$ at $3000 \mathrm{RPM}$. An aliquot of the methanol extract was analyzed with an Agilent 7890 GC equipped with a 5975C MSD. Analyte separation was achieved on an Agilent Cam column $(30 \mathrm{~m} \times 0.250 \mathrm{~mm} \times 0.25 \mu \mathrm{m})$. The limit of detection for nicotine was $0.88 \mu \mathrm{g} / \mathrm{g}$ tobacco.

\section{RESULTS}

Cigarettes were analyzed for the amount of nicotine in the tobacco filler and also for the amount of nicotine in the smoke. "Tar" and carbon monoxide in smoke were also analyzed. The results of the analyses are presented in Table 3. Tobacco filler has varying amounts of water dependent on how the product was manufactured and on the humectants used. Nicotine in the tobacco filler is presented as it was measured ("as is" basis) and then corrected to a dry weight basis. As expected, there was no difference in the nicotine content or yield between VLNTM Kings and VLN ${ }^{\mathrm{TM}}$ Menthol Kings since the only difference between the two products is addition of a menthol solution to the filter. On an "as is" basis the average nicotine in the tobacco filler for the top 100 brand styles in the U.S. was $17.2 \mathrm{mg}$ nicotine $/ \mathrm{g}$ tobacco filler. The amount of nicotine in VLN"M was $0.41 \mathrm{mg} / \mathrm{g}$ tobacco on an "as is" basis. When the results were corrected for variations in the water content, the top 100 brand styles averaged $19.4 \mathrm{mg}$ nicotine/g tobacco filler dry weight (range 14.7-33.2). VLNTM cigarettes averaged $0.47 \mathrm{mg}$ of nicotine $/ \mathrm{g}$ tobacco filler. Figure 1 shows the dramatic differences between VLN ${ }^{\mathrm{TM}}$ products and the top 100 brand styles sold in the U.S. A more relevant comparison is on a cigarette basis. The weight of tobacco varies between commercial cigarettes. On a per-cigarette basis the average nicotine in the tobacco filler for the top 100 brand styles in the U.S. was $12.0 \mathrm{mg}$ nicotine/cig (range 8.2-24.1). The amount of nicotine in $\mathrm{VLN}^{\mathrm{TM}}$ was $0.27 \mathrm{mg} / \mathrm{cig}$. Figure 2 illustrates the differences between VLN ${ }^{\mathrm{TM}}$ products and the top 100 brand styles sold in the U.S. on a per-cigarette basis. The smoke from the cigarettes was also analyzed under ISO smoking conditions to see if design differences might affect the theoretical amount of nicotine delivered to the smoker. The average nicotine yield was $0.903 \mathrm{mg} / \mathrm{cig}$ (range: $0.38-1.82$ ) for the top 100 brand styles. VLNTM cigarettes yielded $0.03 \mathrm{mg}$ nicotine/cig under ISO smoking conditions. 
Table 3. Nicotine content and yield.

\begin{tabular}{|c|c|c|c|c|c|c|}
\hline \multirow[b]{2}{*}{ Product } & \multicolumn{3}{|c|}{ ISO smoke analysis (mg/cig) } & \multicolumn{3}{|c|}{ Filler analysis } \\
\hline & "Tar" & Nicotine & $\mathrm{CO}$ & $\begin{array}{c}\text { Nicotine } \\
(\mathrm{mg} / \mathrm{g})\end{array}$ & $\begin{array}{c}\text { Nicotine } \\
(\mathrm{mg} / \mathrm{g}) \text { dry } \\
\text { weight basis }\end{array}$ & $\begin{array}{l}\text { Nicotine } \\
\text { (mg/cig) }\end{array}$ \\
\hline VLN King & 7.12 & 0.030 & 11.3 & 0.41 & 0.47 & 0.27 \\
\hline VLN Menthol King & 6.90 & 0.030 & 11.0 & 0.41 & 0.47 & 0.27 \\
\hline Camel Blue King & 9.84 & 0.837 & 10.9 & 17.2 & 19.7 & 11.7 \\
\hline Marlboro Gold King & 8.97 & 0.670 & 10.2 & 15.9 & 18.2 & 10.3 \\
\hline Marlboro Menthol Gold King & 9.44 & 0.741 & 9.9 & 16.6 & 19.0 & 10.5 \\
\hline Marlboro Red King & 14.00 & 0.956 & 13.3 & 15.8 & 17.4 & 11.0 \\
\hline Marlboro Special Blend Gold King & 8.87 & 0.675 & 9.9 & 15.9 & 18.1 & 10.2 \\
\hline Newport Menthol Green King & 15.40 & 1.080 & 15.1 & 17.9 & 20.3 & 12.0 \\
\hline Camel 99 Blue & 12.30 & 0.890 & 11.9 & 17.3 & 19.5 & 13.6 \\
\hline Camel 99 Full Flavor Bx & 16.30 & 1.230 & 15.2 & 16.8 & 18.9 & 13.2 \\
\hline Camel Crush King & 11.90 & 0.941 & 12.2 & 17.2 & 19.7 & 10.8 \\
\hline Camel Crush Menthol King & 13.50 & 1.070 & 12.5 & 17.5 & 20.0 & 10.9 \\
\hline Camel Crush Menthol Silver King & 11.90 & 0.916 & 12.1 & 17.0 & 19.2 & 10.9 \\
\hline Camel Full Flavor Filter King & 15.10 & 1.210 & 14.1 & 17.3 & 19.7 & 12.2 \\
\hline Winston White King & 4.50 & 0.383 & 6.8 & 16.1 & 18.3 & 10.4 \\
\hline Newport Menthol Smooth Select 100 & 12.30 & 1.010 & 13.6 & 17.8 & 20.3 & 13.6 \\
\hline Camel Turkish Blend Gold King & 9.64 & 0.727 & 11.5 & 15.6 & 17.4 & 9.3 \\
\hline Camel Turkish Blend Jade Silver King & 9.69 & 0.755 & 10.4 & 16.6 & 18.3 & 9.6 \\
\hline Camel Turkish Blend Royal Filter King & 14.10 & 1.000 & 13.7 & 16.4 & 18.8 & 10.2 \\
\hline Camel Wides Red King & 17.50 & 1.220 & 18.3 & 17.7 & 20.1 & 14.6 \\
\hline Eagle 20'S Red 100 & 14.40 & 0.996 & 16.9 & 17.3 & 19.6 & 12.1 \\
\hline Kool Blue King & 10.10 & 0.762 & 10.5 & 16.8 & 19.2 & 10.5 \\
\hline Kool Green 100 & 13.50 & 1.000 & 14.8 & 17.1 & 19.6 & 12.3 \\
\hline Kool Menthol King & 14.80 & 1.050 & 14.1 & 17.0 & 19.4 & 11.3 \\
\hline L\&M 100 & 11.70 & 0.707 & 14.5 & 13.2 & 14.7 & 9.3 \\
\hline L\&M 100 Menthol & 14.10 & 1.020 & 16.5 & 17.5 & 20.0 & 12.5 \\
\hline L\&M Blue 100 & 9.11 & 0.599 & 13.0 & 13.9 & 15.9 & 9.8 \\
\hline L\&M Blue King & 9.15 & 0.584 & 12.1 & 13.9 & 15.7 & 8.2 \\
\hline L\&M Bold Menthol 100 & 17.20 & 1.220 & 16.7 & 17.7 & 20.1 & 13.2 \\
\hline L\&M Full Flavor King & 12.10 & 0.678 & 13.0 & 13.5 & 15.2 & 9.0 \\
\hline L\&M Menthol King & 13.60 & 0.973 & 13.7 & 18.6 & 21.2 & 12.5 \\
\hline Marlboro 72 Gold & 9.12 & 0.675 & 9.6 & 16.8 & 19.2 & 9.8 \\
\hline Marlboro Menthol Blue King & 11.20 & 0.802 & 12.1 & 17.8 & 20.3 & 10.9 \\
\hline Marlboro 72 Menthol Green & 14.00 & 0.863 & 13.3 & 17.7 & 20.3 & 10.0 \\
\hline Marlboro 72 Red & 12.10 & 0.812 & 11.6 & 15.7 & 17.9 & 9.3 \\
\hline Marlboro 72 Silver & 5.34 & 0.488 & 6.3 & 20.2 & 23.0 & 10.2 \\
\hline Marlboro 83 & 14.20 & 0.985 & 13.3 & 16.4 & 18.7 & 11.5 \\
\hline Marlboro Black Menthol 100 & 16.00 & 1.100 & 16.3 & 16.6 & 18.8 & 11.8 \\
\hline Marlboro Black Menthol King & 15.10 & 0.959 & 14.4 & 16.2 & 18.6 & 10.1 \\
\hline Marlboro Blend 27 King & 11.90 & 0.829 & 12.7 & 16.0 & 18.0 & 9.8 \\
\hline Marlboro Edge King & 15.00 & 0.987 & 14.4 & 16.2 & 18.5 & 11.0 \\
\hline Marlboro Gold 100 & 8.72 & 0.679 & 10.8 & 16.2 & 18.5 & 11.9 \\
\hline Marlboro Green (Menthol) King & 14.40 & 0.980 & 13.5 & 16.9 & 18.9 & 11.8 \\
\hline Marlboro Menthol 100 & 14.50 & 1.020 & 15.6 & 17.3 & 19.7 & 13.3 \\
\hline Marlboro Menthol Gold 100 & 9.09 & 0.731 & 11.3 & 17.4 & 19.8 & 12.9 \\
\hline Marlboro Midnight Menthol King & 15.50 & 0.980 & 14.0 & 16.4 & 18.3 & 10.7 \\
\hline Marlboro Nxt King & 8.08 & 0.664 & 7.8 & 16.3 & 18.2 & 9.4 \\
\hline Marlboro Red 100 & 15.30 & 1.090 & 15.4 & 16.7 & 18.8 & 12.8 \\
\hline Marlboro Red Label 100 & 13.10 & 0.952 & 13.5 & 16.5 & 18.8 & 12.0 \\
\hline Marlboro Red Label King & 14.40 & 0.978 & 13.5 & 16.4 & 18.7 & 10.9 \\
\hline Marlboro Silver 100 & 4.50 & 0.404 & 6.5 & 16.9 & 19.3 & 11.6 \\
\hline Marlboro Silver King & 4.97 & 0.424 & 6.5 & 17.3 & 19.7 & 10.2 \\
\hline Marlboro Silver Menthol 100 & 5.91 & 0.493 & 8.3 & 17.1 & 19.4 & 12.3 \\
\hline Marlboro Silver Menthol King & 5.13 & 0.427 & 6.7 & 16.9 & 18.9 & 10.3 \\
\hline Marlboro Smooth Menthol 100 & 13.00 & 0.952 & 14.4 & 17.7 & 20.2 & 12.7 \\
\hline Marlboro Smooth Menthol King & 14.20 & 0.936 & 14.2 & 17.4 & 19.9 & 10.4 \\
\hline Marlboro Southern Cut King & 15.50 & 0.995 & 13.9 & 16.4 & 18.5 & 10.8 \\
\hline
\end{tabular}




\begin{tabular}{|c|c|c|c|c|c|c|}
\hline \multirow[b]{2}{*}{ Product } & \multicolumn{3}{|c|}{ ISO smoke analysis (mg/cig) } & \multicolumn{3}{|c|}{ Filler analysis } \\
\hline & "Tar" & Nicotine & $\mathrm{CO}$ & $\begin{array}{c}\text { Nicotine } \\
(\mathrm{mg} / \mathrm{g})\end{array}$ & \begin{tabular}{|c|} 
Nicotine \\
$(\mathrm{mg} / \mathrm{g})$ dry \\
weight basis
\end{tabular} & $\begin{array}{l}\text { Nicotine } \\
\text { (mg/cig) }\end{array}$ \\
\hline Marlboro Special Blend Black 100 & 14.80 & 1.000 & 15.3 & 16.8 & 19.0 & 12.2 \\
\hline Marlboro Special Blend Black King & 14.60 & 0.938 & 14.1 & 16.7 & 18.5 & 10.3 \\
\hline Marlboro Special Blend Black Menthol 100 & 15.40 & 0.994 & 16.0 & 16.3 & 18.5 & 11.8 \\
\hline Marlboro Special Blend Black Menthol King & 15.20 & 0.978 & 14.0 & 16.9 & 19.3 & 10.5 \\
\hline Marlboro Special Blend Gold 100 & 9.90 & 0.766 & 12.3 & 17.0 & 19.4 & 12.6 \\
\hline Marlboro Special Blend Red 100 & 12.80 & 0.892 & 13.5 & 16.4 & 18.6 & 12.0 \\
\hline Marlboro Special Blend Red King & 10.80 & 0.750 & 11.1 & 16.3 & 18.4 & 10.1 \\
\hline Maverick Full Flavor 100 & 14.20 & 1.000 & 15.3 & 15.2 & 17.3 & 12.2 \\
\hline Maverick Gold 100 & 7.76 & 0.584 & 9.4 & 15.5 & 17.7 & 12.2 \\
\hline Maverick Menthol 100 & 16.60 & 1.160 & 16.2 & 15.7 & 17.8 & 13.2 \\
\hline Misty 120 Blue & 11.60 & 1.020 & 10.8 & 16.2 & 18.4 & 14.0 \\
\hline Misty 120 Menthol (Green) & 10.20 & 0.809 & 10.1 & 14.8 & 16.9 & 12.4 \\
\hline Natural American Spirit Blue King & 17.00 & 1.820 & 13.0 & 21.9 & 24.8 & 18.6 \\
\hline Natural American Spirit Menthol Green King & 16.40 & 1.780 & 12.8 & 24.3 & 27.2 & 20.2 \\
\hline Natural American Spirit Organic Mellow Gold King & 7.72 & 1.110 & 9.7 & 29.3 & 33.2 & 23.8 \\
\hline Natural American Spirit Organic Turquoise King & 14.00 & 1.820 & 12.0 & 28.9 & 32.6 & 24.1 \\
\hline Natural American Spirit Yellow Mellow King & 13.10 & 1.410 & 11.5 & 22.6 & 25.6 & 18.9 \\
\hline Newport Menthol Gold 100 & 12.00 & 0.889 & 12.0 & 17.8 & 19.8 & 14.2 \\
\hline Newport Menthol Gold King & 8.38 & 0.662 & 9.2 & 18.6 & 21.2 & 11.4 \\
\hline Newport Menthol Green 100 & 18.20 & 1.500 & 15.5 & 18.9 & 21.4 & 14.8 \\
\hline Newport Non-Menthol 100 & 18.80 & 1.490 & 15.9 & 18.8 & 21.4 & 15.0 \\
\hline Newport Non-Menthol King & 14.90 & 1.050 & 15.2 & 18.3 & 20.7 & 11.8 \\
\hline Pall Mall Black Menthol 100 & 14.50 & 1.110 & 14.2 & 17.6 & 19.6 & 12.4 \\
\hline Pall Mall Blue 100 & 10.20 & 0.854 & 10.3 & 16.0 & 18.2 & 12.8 \\
\hline Pall Mall Blue King & 10.20 & 0.828 & 9.9 & 16.3 & 18.5 & 11.0 \\
\hline Pall Mall Menthol 100 & 10.40 & 0.838 & 11.0 & 15.9 & 18.0 & 13.0 \\
\hline Pall Mall Menthol King & 10.40 & 0.819 & 10.9 & 15.9 & 17.9 & 10.9 \\
\hline Pall Mall Orange 100 & 5.04 & 0.438 & 7.0 & 15.8 & 17.8 & 12.9 \\
\hline Pall Mall Orange 85 & 5.01 & 0.435 & 7.1 & 16.0 & 18.1 & 10.7 \\
\hline Pall Mall Red 100 & 14.10 & 1.090 & 13.2 & 16.1 & 18.3 & 14.1 \\
\hline Pall Mall Red King & 13.80 & 1.080 & 11.3 & 16.0 & 18.1 & 12.3 \\
\hline Pall Mall White Menthol 100 & 7.89 & 0.661 & 9.5 & 18.5 & 21.0 & 13.4 \\
\hline Parliament White King & 8.40 & 0.579 & 9.7 & 16.8 & 19.1 & 9.8 \\
\hline Pyramid Blue 100 & 8.90 & 0.693 & 11.3 & 15.8 & 17.1 & 10.8 \\
\hline Pyramid Red 100 & 14.50 & 0.971 & 15.7 & 17.2 & 19.4 & 12.1 \\
\hline Salem Green King & 15.70 & 0.958 & 15.5 & 16.1 & 18.2 & 11.1 \\
\hline Salem Menthol Gold King & 8.14 & 0.595 & 10.8 & 16.2 & 18.4 & 10.1 \\
\hline Salem Slim Menthol 100 & 6.92 & 0.570 & 7.9 & 15.9 & 18.0 & 10.1 \\
\hline USA Gold King & 10.40 & 0.699 & 11.7 & 17.7 & 19.8 & 9.4 \\
\hline USA Gold Red 100 & 14.20 & 1.030 & 13.9 & 18.7 & 21.2 & 11.9 \\
\hline USA Gold Red King & 12.50 & 0.902 & 11.8 & 18.1 & 20.6 & 10.3 \\
\hline Winston Gold 100 & 9.19 & 0.757 & 12.8 & 16.8 & 19.1 & 12.3 \\
\hline Winston Gold King & 9.33 & 0.760 & 11.1 & 17.2 & 19.5 & 10.9 \\
\hline Winston Red 100 & 13.70 & 1.100 & 15.0 & 17.7 & 19.3 & 13.0 \\
\hline Winston Red King & 12.90 & 0.983 & 13.6 & 17.2 & 19.6 & 12.3 \\
\hline Mean & 11.96 & 0.903 & 12.37 & 17.2 & 19.4 & 12.0 \\
\hline SD & 3.38 & 0.276 & 2.66 & 2.3 & 2.6 & 2.6 \\
\hline Min & 4.50 & 0.380 & 6.26 & 13.20 & 14.70 & 8.20 \\
\hline Max & 18.80 & 1.820 & 18.30 & 29.30 & 33.20 & 24.10 \\
\hline VLN King & 7.12 & 0.030 & 11.3 & 0.41 & 0.47 & 0.27 \\
\hline VLN Menthol King & 6.90 & 0.030 & 11.0 & 0.41 & 0.47 & 0.27 \\
\hline
\end{tabular}

Figure 3 graphically presents the differences between VLN $^{\mathrm{TM}}$ cigarettes and the top brand styles. On a percigarette basis VLNTM cigarettes contained $0.27 \mathrm{mg}$ of nicotine in the tobacco and yielded $0.03 \mathrm{mg}$ of nicotine in the smoke, a conversion efficiency of $11.1 \%$. The commer- cial cigarettes contained on average $12 \mathrm{mg}$ of nicotine per cigarette and yielded $0.903 \mathrm{mg}$ of nicotine in smoke, a conversion efficiency of $7.5 \%$. These differences in efficiency are not unexpected since yield is dependent on the amount of tobacco, the type of filter, and the amount of 
Natural American Spirit Organic Mellow Gold King Natural American Spirit Organic Turquoise King Natural American Spirit Menthol Green King Natura American Spint Yellow Mellow King

Natural Am Manboro 72 Silver Newport Non-Menthol100 USA Gold Red 100 L\&M Menthol King Newport Menthol Gold King Newport Non-Menthol King USA Gold Red King Newport Menthol Green King Marlboro 72 Menthol Green Newport Menthol Smooth Select 100 Marlboro Smooth Menthol100 Camel Wides Red King L8M 100 Menthol Camel Crush Menthol King Marlboro Smooth Menthol King Newport Menthol Gold 100 Mariboro USA Gold King Menthol Gold 100 Camel Filter King Camel Crush King Camel Blue King

Pall Mall Black Menthol100 Eagle 20'S Red 100 Winston Red King Camel 99 Blue Winston Gold King Kool Menthol King Pyramid Red 100

Marlboro Special Blend Gold 100 Marlboro Special Blend Black Menthol King Winston Red 100

Camel Crush Menthol Silver King Kool Blue King Marlboro 72 Gold Winston Gold 100 Parliament White King Marlboro Special Blend Black 100 Marlboro Menthol Gold King
Camel9g Marlboro Green King Marlboro Silver Menthol King Mariboro Black Menthol100 Camel Turkish Blend Royal Filter King Marlboro Red Label 100

Marlboro Red Label King

Mariboro Black Menthol King

Marlboro Special Blend Red 100 Marlboro Sporo Southern Cut King Marlboro Edge King cial Blend Black King Pall Mall Blue King Misty 120 Blue

Marlboro Special Blend Red King Salem Menthol Gold King Marlboro Midnight Menthol King
Pall Mall Red 100 Camel Turkish Blend Jade Silver King
Winston White King Salem Green King Pall Mall Blue 100 Marlboro Nxt King Pall Mall Red King Marlboro Special Blend Gold King
Pall Mall Orange 85 Pall Mall Orange 85

Pall Mall Menthol 100 Sale $m$ Slim Menthol 100 Marlboro 72 Red Pall Mall Menthol King

Maverick Menthol 100

Pall Mall Orange 100 Maverick Gold 100 Camel Turkish Blend Gold King Maverick 100 Pyramid Blue 100 Misty 120 Mentho L\&M Blue 100 L\&M Blue King
Full Flavor King

L\&M Full Flavor King

L\&M 100

VLN Menthol $: 0.5$
VLN Regular 0.5
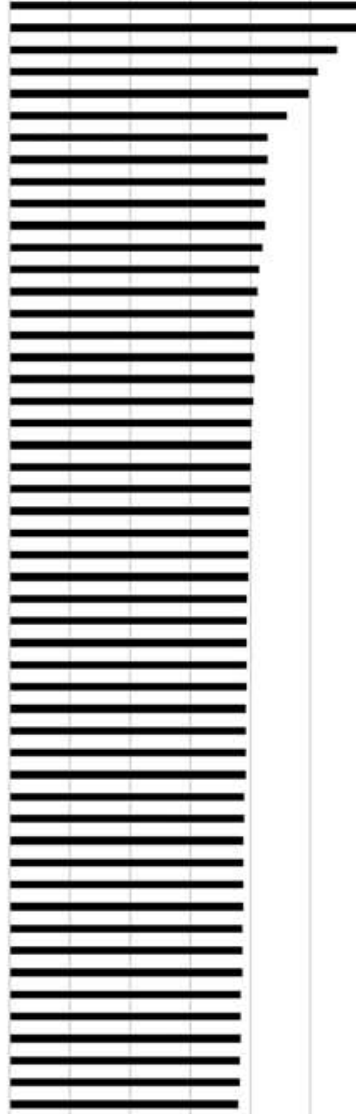

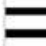
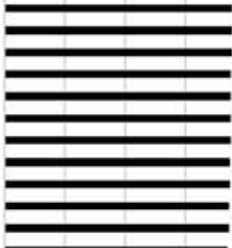

$$
\text { - }
$$$$
\text { 吾要 }
$$

$$
\text { 原 }
$$
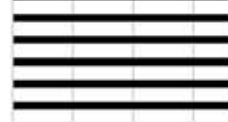

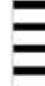
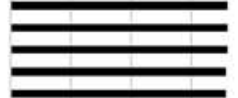

$$
\text { 三 }
$$
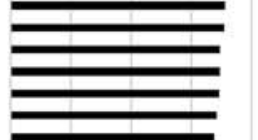

$$
\text { 原事 }
$$$$
\text { : }
$$

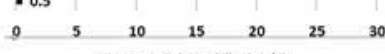

Nicotine in Tobacco Filler (me/s)

Figure 1. Comparison of nicotine content in tobacco filler on a dry weight basis. 
Natural American Spirit Organic Turquoise King Natural American Spirit Organic Mellow Gold King Natural American Spirt Menthol Light Green King Natural American Spirit Yellow Mellow King Natural American Spirit Blue King Newport Non-Menthol 100 Camel Wides Red King Camel Wides Red King Pall Mall Red 100 Misty 120 Blue
Newport Menthol Smooth Select 100
Camel 99 Blue

Pall Mall White Menthol 100 Maverick Menthol 100 L\&M Bold Menthol 100 inston Red 100 Winston Red 100
Pall Mall Menthol 100 Pall Mall Orange Too Marlboro Menthol Gold 100 Pall Mall Blue 100 Marlboro Smooth Menthol 100 Mariboro Special Blend Gold 100 L\&M Menthol King L\&M 100 Mentho

Pall Mall Black 120 Mentho Misty 120 Mentho Winston Red King Winston Gold 100

Marlboro Silver Menthol 100 averick Gold 100 Maverick 100

Marlboro Special Blend Black 100 Camel Filter King Pyramid Red 100 Newport Menthol Green Kin Newport Menthol Green King
Marlboro Special Blend Red 100

riboro Red Label 100 USA Gold Red 100

Newport Non-Menthol King

Marlboro Special Blend Black Menthol100 Marlboro Green King

Marlboro Black Menthol100 Camel Blue King Marlboro 83

Newport Menthol Gold King Kool Menthol King Salem Green King Marlboro Red King Pall Mall Blue King Marlboro Edge King Winston Gold King Pall Mall Menthol King Marlboro Red Label King Camel Crush Menthol Sliver King Camel Crush Menthol King Pyramid Blue 100 Camel Crush King
Cam

Marlboro Midnight Menthol King Marlboro Special Blend Black Menthol King Kool Blue King

Marlboro Smooth Menthol King Winston White King Mariboro Gold King USA Gold Red King Marlboro Special Blend Black King Marlboro Silver Menthol King

Marlboro Special Blend Gold King
Marlboro Silver King Marlboro 72 Silver Camel Turkish Blend Royal Filter King Salem Menthol Gold King

Marlboro Special Blend Red King

Marlboro 72 Menthol Green Parliament White King Marlboro Blend 27 King Marlboro 72 Gold
L\&M Blue 100 Camel Turkish Blend Jade Silver King Marlboro Nxt King Marlboro 72 Red

Camel Turkish Blend Gold King L\&M Full Flavor King LaM Blue King VIN King
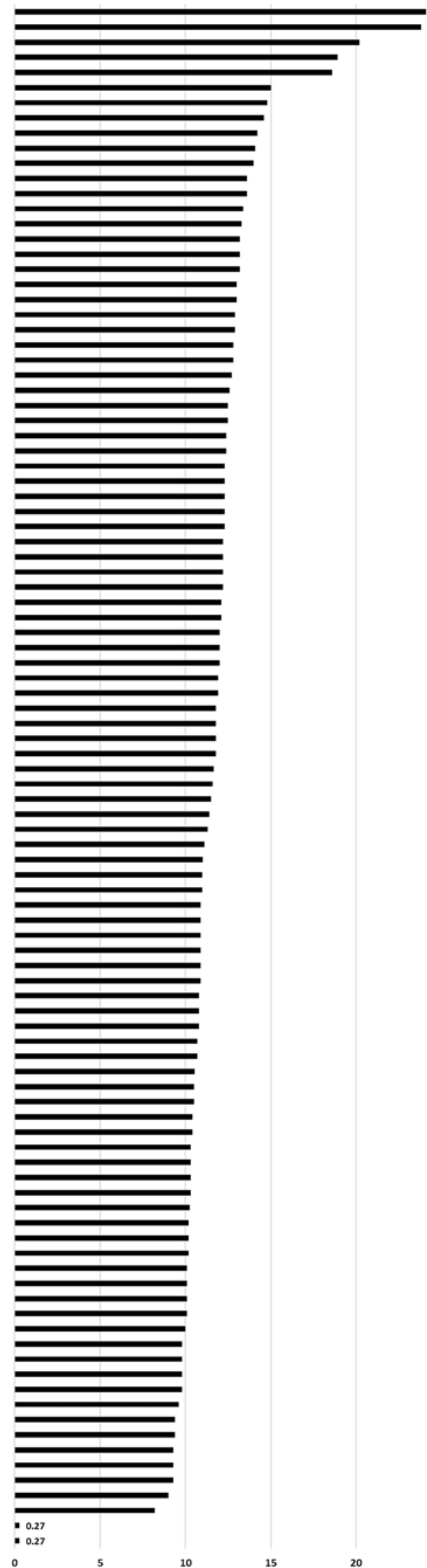

Nicotine in Clgarette $(\mathrm{mg} / \mathrm{cig})$

Figure 2. Comparison of nicotine content on a per-cigarette basis. 


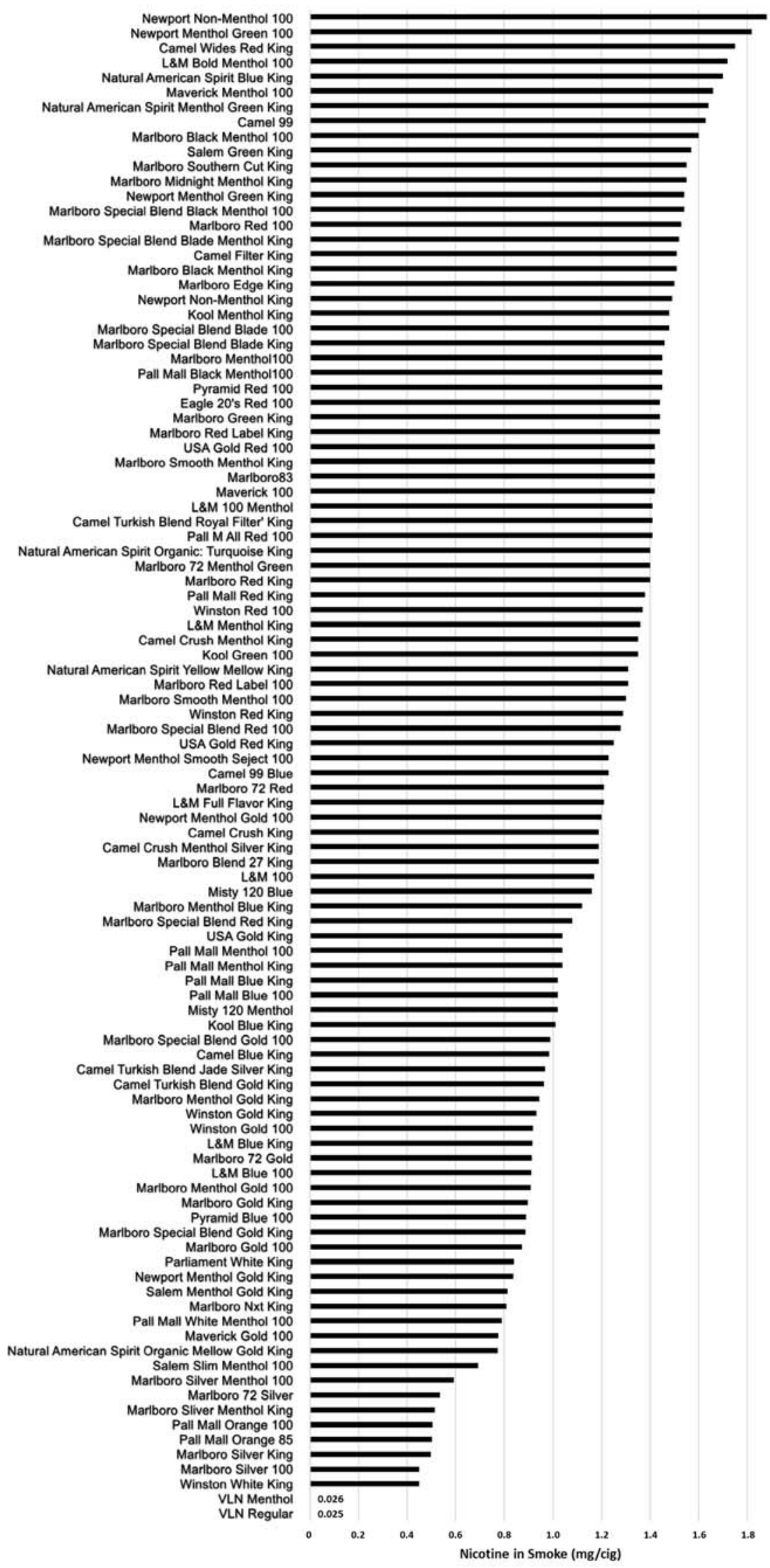

Figure 3. Comparison of nicotine in smoke on a per-cigarette basis. 
ventilation. VLN ${ }^{\mathrm{TM}}$ cigarettes contain about $650 \mathrm{mg}$ of tobacco which is consistent with the amount of tobacco normally used in conventional king size cigarettes. Some of the marketed cigarettes in the top 100 list were 100 ' $^{2}$ which generally contain more tobacco and generally have slightly higher "tar" yields on a per-cigarette basis. Most commercial filter cigarettes in the U.S. use a single cellulose acetate filter with or without added carbon. The filter used in VLNTM was a dual filter made up of a cellulose acetate segment on the mouth end and a crepe paper filter on the tobacco end. This different filter design and different degrees of ventilation (ventilation was not measured in this study for the commercial cigarettes) may account for some of the differences in the nicotine yield.

"Tar" and carbon monoxide were also measured in the cigarette smoke. The average "tar" yield in smoke for the top brand styles was $11.96 \mathrm{mg} / \mathrm{cig}$ (range 4.5-18.0). The average "tar" yield for VLNTM was $7 \mathrm{mg} / \mathrm{cig}$. "Tar" yield is dependent on the amount of tobacco, the type of filter, and the amount of ventilation which may be different between VLNTM cigarettes and the top brands. The average $\mathrm{CO}$ yield for the top brands was $12.4 \mathrm{mg} / \mathrm{cig}$ (range 6.3-18.3). The average CO yield for VLNTM was $11.2 \mathrm{mg} / \mathrm{cig}$. The amount of $\mathrm{CO}$ in smoke is determined mostly by the amount of ventilation in the tipping paper. ${ }^{3}$ VLN ${ }^{\mathrm{TM}}$ uses a tipping paper with about $13 \%$ ventilation. The amount of ventilation in commercial cigarettes can range from 0 to $70 \%$ (18).

\section{DISCUSSION}

VLNTM cigarettes are substantially lower in nicotine than any other cigarettes currently sold to consumers in the United States - whether measured on a cigarette, tobacco dry weight, or cigarette smoke yield basis. VLNTM cigarettes contain a target level of $0.5 \mathrm{mg}$ of nicotine $/ \mathrm{g}$ of tobacco filler (dry weight). The actual measured amount was $0.47 \mathrm{mg}$ of nicotine $/ \mathrm{g}$ of tobacco. Analysis of the top 100 leading cigarette brands in the U.S. showed that, on average, these cigarettes contained $19.4 \mathrm{mg}$ of nicotine/g of tobacco on a dry weight basis. Accordingly, the nicotine content of VLNTM cigarette tobacco is $97.6 \%$ lower than the average nicotine content of the tobacco filler in the top 100 leading brands. When compared to the lowest nicotine content product of any of the top 100 leading US brands, "L\&M 100", the nicotine content of VLNTM is $96 \%$ lower. When compared on a cigarette basis, the top 100 brands average $12.0 \mathrm{mg}$ of nicotine and VLN ${ }^{\mathrm{TM}}$ averages $0.27 \mathrm{mg}$. Therefore, without exception, VLNTM cigarettes contain at least $95 \%$ less nicotine than the top 100 brands in the U.S.. Similarly, the amount of nicotine in smoke (yield) for VLNTM cigarettes is $0.03 \mathrm{mg} / \mathrm{cig}$, which is also $97 \%$ lower than the top 100 cigarette brand style's nicotine smoke yields $(0.903 \mathrm{mg} / \mathrm{cig})$. Because of the unique technology

2

King size cigarettes are generally considered to be about $85 \mathrm{~mm}$ long and 100's are about $100 \mathrm{~mm}$ long. While these terms are used as general indicators of the length of the cigarettes, manufacturers may make cigarettes with slightly different lengths that are sold as belonging to either the category "Kings" or "100's".

3 The focus of this research was the nicotine content and nicotine yield of the cigarettes. Ventilation was not measured. used to reduce nicotine and the absence of the technology in any other commercial cigarette on the market, it is believed that the nicotine level in VLN ${ }^{\mathrm{TM}}$ is reduced by at least $95 \%$ when compared to all other cigarettes available in the U.S. marketplace irrespective of the comparison basis.

Clinical studies indicate that use of VLN ${ }^{\mathrm{TM}}$ cigarettes can lead to substantial reductions in nicotine exposure (19-23) as a result of the lower nicotine delivery and also due to an overall reduction in cigarettes consumed per day $(20$, 23-27). In the longest (20 weeks) and largest $(1,250$ subjects with no intention to quit) study to date on SPECTRUM ${ }^{\circledR} / V^{2} N^{T M}$ cigarettes, HATSUKAMI et al. observed more than a $50 \%$-reduction in cigarettes per day (28). The "gold standard" for measuring reduced nicotine exposure is the amount of total nicotine equivalents (TNE) in the urine. HATSUKAMI et al. observed a $57 \%$ reduction in TNE in the 20-week study with SPECTRUM®/VLNTM cigarettes. There is clear evidence that consumption of cigarettes that have very low levels of nicotine in the tobacco will result in reductions in the nicotine in the smoke and the smoker will be exposed to significantly lower levels of nicotine as indicated by the total nicotine equivalents in the urine. This is due to reduced nicotine intake and cigarette consumption over the duration of use. KAMENS et al.(29) compared the pharmacokinetic profile of SPECTRUM $\AA / V L N N^{T M}$ cigarettes to usual brand after a single use in twelve daily cigarette smokers. Nicotine area under the curve was reduced by $95 \%$. In most clinical studies with VLNTM there is a tendency for subjects to "cheat" and use non-study cigarettes (30-32). This probably accounts for the only $57 \%$ reduction in TNE observed by HATSUKAMI et al. after 20 weeks of use compared to the $95 \%$ reduction in nicotine plasma levels after single use under confined circumstances. There is very little nicotine in VLNTM cigarettes and compensation has not been observed after long term use $(19,20,21,23,28)$.

Premarket Tobacco Applications (PMTA) and Modified Risk Tobacco Applications (MRTPA) were submitted to the United States Food and Drug Administration for VLNTM cigarettes in December of 2018. Section 911(h)(2)(B) of the Tobacco Control Act states that the Secretary may require that the percent (or fraction) of change and identity of the reference tobacco product and a quantitative comparison of the amount of the substance claimed to be reduced shall be stated in immediate proximity to the most prominent claim. The principal reduced exposure claim for the VLNTM King and VLNTM Menthol King products will be " $95 \%$ Less Nicotine". This claim is based on the nicotine content of the filler in the cigarette irrespective of the basis of comparison (per $g$ of tobacco filler, per $g$ of tobacco filler calculated on a dry weight basis, or per cigarette). The tobacco filler target nicotine level is $0.5 \mathrm{mg}$ nicotine/g of tobacco filler (dry weight). The top 100 brand styles in the U.S. were identified and analyzed. These results demonstrated that VLNTM cigarettes had reduced nicotine levels of $95 \%$ less in the tobacco filler and smoke compared to the top 100 brands representing over $80 \%$ of all cigarettes sold in the U.S. Because of the unique technology used to reduce nicotine and the absence of the technology in any other cigarette on the market, it is believed that the nicotine level in VLNTM is 
reduced by at least $95 \%$ when compared to all other cigarettes in the marketplace and a comparator statement such as "leading brands", "usual brand", "typical brands", or "top three brands" is not required. Analysis of the smoke yield of VLN VM cigarettes also demonstrated that the amount of nicotine in smoke was reduced at least $95 \%$ also.

\section{ACKNOWLEDGEMENTS}

We would like to acknowledge Karen Carter for sourcing the 100 different brand styles tested in this study. We would also like to thank the technical team at Enthalpy Analytical. This research was funded by 22 nd Century Group. Inc.

\section{REFERENCES}

1. Benowitz, N.L. and J.E. Henningfield: Establishing a Nicotine Threshold for Addiction. The Implications for Tobacco Regulation; N. Engl. J. Med. 331 (1994) 123-125. DOI: 10.1056/NEJM199407143310212

2. Federal Drug Association (FDA), Commissioner of the FDA: Press Announcements - FDA Announces Comprehensive Regulatory Plan to Shift Trajectory of Tobacco-related Disease, Death; FDA News Release July 28,2017. Available at: https://www.fda.gov/newsevents/press-announcements/fda-announcescomprehensive-regulatory-plan-shift-trajectory-tobaccorelated-disease-death (accessed June 28, 2019).

3. Layton, L: FDA Should Use its Power to Lower Nicotine in Cigarettes, Former Chief Says; Washington Post June 17, 2010. Available at http://www.washingtonpost. com/wp-dyn/content/article/2010/06/16/AR201006 1605340.html?noredirect=on (accessed June 28, 2019).

4. Federal Drug Association (FDA), Commissioner of the FDA: Speeches by FDA Officials - Protecting American Families: Comprehensive Approach to Nicotine and Tobacco; Speech June 28, 2017. Available at: https:// www.fda.gov/news-events/speeches-fda-officials/pro tecting-american-families-comprehensive-approachnicotine-and-tobacco-06282017 (accessed June 28, 2019).

5. Food and Drug Administration (FDA): Tobacco Product Standard for Nicotine Level of Combusted Cigarettes; 83 Fed. Reg. 11818 (2018). Available at: https://www.federalregister.gov/d/2018-05345 (accessed July 25, 2019).

6. National Institute on Drug Abuse (NIDA): NOT-DA14-004: Notice of Availability of Nicotine Research Cigarettes through NIDA's Drug Supply Program; available at: https:/grants.nih.gov/grants/guide/noticefiles/NOT-DA-14-004.html (accessed June 28,2019).

7. Becker, K.M., J.E. Rose, and A.P. Albino: A Randomized Trial of Nicotine Replacement Therapy in Combination with Reduced-Nicotine Cigarettes for Smoking Cessation; Nicotine Tob. Res. 10 (2008) 1139-1148. DOI: $10.1080 / 14622200802123294$

8. International Organisation for Standardization (ISO): ISO 3402:1999 Tobacco and Tobacco Products Atmosphere for Conditioning and Testing; ISO,
Geneva, Switzerland, 1999. Available at: https://www. iso.org/obp/ui/\#iso:std:iso:3402:ed-4:v1:en (accessed July 25, 2019).

9. International Organisation for Standardization (ISO): ISO 10362-1:1999 Cigarettes - Determination of Water in Smoke Condensates - Part 1: Gas-Chromatographic Method; ISO, Geneva, Switzerland, 1999. Available at: https://www.iso.org/standard/28322.html (accessed July 25, 2019).

10. International Organisation for Standardization (ISO): ISO 8454:2007 Cigarettes - Determination of Carbon Monoxide in the Vapor Phase of Cigarette Smoke NDIR Method; ISO, Geneva, Switzerland, 2007. Available at: https://www.iso.org/obp/ui/\#iso:std:iso: 8454:ed-3:v1:en (accessed July 25, 2019).

11. International Organisation for Standardization (ISO): ISO 3308:2012 Routine Analytical Cigarette-Smoking Machine - Definitions and Standard Conditions; ISO, Geneva, Switzerland, 2012. Available at: https://www.iso.org/obp/ui/\#iso:std:iso:3308:ed-5:v1:en (accessed July 25, 2019).

12. International Organisation for Standardization (ISO): ISO 10315:2013 Cigarettes - Determination of Nicotine in Smoke Condensates - Gas-Chromatographic Method; ISO, Geneva, Switzerland, 2013. Available at: https://www.iso.org/obp/ui/\#iso:std:iso:10315:ed3:v2:en (accessed July 25, 2019).

13. Brown, A.P., H. Liu, K. Manson, M.S. Melvin, J. Pierotti, Y.B. Pithawalla, J.H. Smith, K. Stutt, and K.A. Wagner: Modification of Standardized Methods for the Measurement of Nicotine in Very Low Nicotine Content Cigarettes; Presented at the CORESTA Congress October 22-28, 2018 in Kunming, China. ST 29.

14. American Association for Laboratory Accreditation: A2LA. Scope of Accreditation to ISO/IEC 17025:2005; Cert. No. 1873.01.2019.

15. American Association for Laboratory Accreditation: A2LA. Scope of Accreditation to ISO/IEC 17025:2005; Cert. No. 3198-01.2019.

16. Department of Health and Human Services (DHHS), Centers for Disease Control and Prevention (CDC): Notice Regarding Revisions to the Laboratory Protocol to Measure the Quantity of Nicotine Contained in Smokeless Tobacco Products Manufactured, Imported, or Packaged in the United States; 74 Fed. Reg. 712 (2009). Available at: https://www.govinfo.gov/app/ details/FR-2009-01-07/E9-19 (accessed July 25, 2019).

17. Cooperation Center for Scientific Research Relative to Tobacco (CORESTA): Recommended Method 87: Determination of Nicotine in Tobacco Products by GCMS, April 2018. Available at: https://www.coresta.org/ sites/default/files/technical_documents/main/CRM_87April2018.pdf (accessed June 28, 2019).

18. Norman, V.: The Effect of Perforated Tipping Paper on the Yield of Various Smoke Components. Beitr. Tabakforsch. 7 (1974) 282-287. DOI: 10.2478/cttr-2013-0343

19. Denlinger, R.L., T.T. Smith, S.E. Murphy, J.S. Koopmeiners, N.L. Benowitz, D.K. Hatsukami, L.R. Pacek, C. Colino, S.N. Cwalina, and E.C. Donny: Nicotine and Anatabine Exposure from Very Low Nicotine Content Cigarettes; Tob. Regul. Sci. 2 (2016) 186-203.

DOI: 10.18001/TRS.2.2.9 
20. Donny, E.C., R.L. Denlinger, J.W. Tidey, J.S. Koopmeiners, N.L. Benowitz, R.G. Vandrey, M. al'Absi, S.G. Carmella, P.M. Cinciripini, S.S. Dermody, D.J. Drobes, S.S. Hecht, J. Jensen, T. Lane, C.T. Le, F.J. McClernon, I.D. Montoya, S.E. Murphy, J.D. Robinson, M.L. Stitzer, A.A. Strasser, H. Tindle, and D.K. Hatsukami: Randomized Trial of Reduced-Nicotine Standards for Cigarettes; N. Engl. J. Med. 373 (2015) 1340-1349. DOI: 10.1056/NEJMsa1502403

21. Hatsukami, D.K., S.J. Heishman, R. Isaksson Vogel, R.L. Denlinger, A.N. Roper-Batker, K.M. Mackowick, J. Jensen, S.E. Murphy, B.F. Thomas, and E. Donny: Dose-Response Effects of Spectrum Research Cigarettes; Nicotine Tob. Res. 15 (2013) 1113-1121.

DOI: $10.1093 / \mathrm{ntr} / \mathrm{nts} 247$

22. Hatsukami, D.K., X. Luo, L. Dick, M. Kangkum, S.S. Allen, S.E. Murphy, S.S. Hecht, P.G. Shields, and M. al'Absi: Reduced Nicotine Content Cigarettes and Use of Alternative Nicotine Products: Exploratory Trial; Addiction 112 (2017) 156-167.

DOI: $10.1111 /$ add.13603

23. Pacek, L.R., R. Vandrey, S.S. Dermody, R.L. Denlinger-Apte, A. Lemieux, J.W. Tidey, F.J. McClernon, A.S. Bangdiwala, D.J. Drobes, M. al'Absi, A.A. Strasser, J.S. Koopmeiners, D.K. Hatsukami, and E.C. Donny: Evaluation of a Reduced Nicotine Product Standard: Moderating Effects of and Impact on Cannabis Use; Drug Alcohol Depend. 167 (2016) 228-232. DOI: $10.1016 /$ j.drugalcdep.2016.08.620

24. Foulds, J., A. Hobkirk, E. Wasserman, J. Richie, S. Veldheer, N.M. Krebs, L. Reinhart, and J. Muscat: Estimation of Compliance with Exclusive Smoking of Very Low Nicotine Content Cigarettes Using Plasma Cotinine; Prev. Med. 117 (2018) 24-29.

DOI: 10.1016/j.ypmed.2018.04.011

25. Robinson, J.D., G. Kypriotakis, M. Karam-Hage, C.E. Green, D.K. Hatsukami, P.M. Cinciripini, and E.C. Donny: Cigarette Nicotine Content as a Moderator of the Relationship Between Negative Affect and Smoking; Nicotine Tob. Res. 19 (2017) 1080-1086. DOI: $10.1093 / \mathrm{ntr} / \mathrm{ntx} 068$

26. Shiffman, S., B.F. Kurland, S.M. Scholl, and J.M. Mao: Nondaily Smokers' Changes in Cigarette Consumption with Very Low-Nicotine-Content Cigarettes: A Randomized Double-Blind Clinical Trial; JAMA Psychiatry 75 (2018) 995-1002.

DOI:10.1001/jamapsychiatry.2018.1831

27. Tidey, J.W., L.R. Pacek, J.S. Koopmeiners, R. Vandrey, N. Nardone, D.J. Drobes, N.L. Benowitz, S.S. Dermody, A. Lemieux, R.L. Denlinger, R. Cassidy, M. al'Absi, D.K. Hatsukami, and E.C. Donny: Effects of 6-Week Use of Reduced-Nicotine Content Cigarettes in Smokers with and Without Elevated Depressive Symptoms; Nicotine Tob. Res. 19 (2017) 59-67.

DOI: $10.1093 /$ ntr/ntw199
28. Hatsukami, D.K., X. Luo, J.A. Jensen, M. al'Absi, S.S. Allen, S.G. Carmella, M. Chen, P.M. Cinciripini, R. Denlinger-Apte, D.J. Drobes, J.S. Koopmeiners, T. Lane, C.T. Le, S. Leischow, K. Luo, F.J. McClernon, S.E. Murphy, V. Paiano, J.D. Robinson, H. Severson, C. Sipe, A.A. Strasser, L.G. Strayer, M.K. Tang, R. Vandrey, S.S. Hecht, N.L. Benowitz, and E.C. Donny: Effect of Immediate vs Gradual Reduction in Nicotine Content of Cigarettes on Biomarkers of Smoke Exposure: A Randomized Clinical Trial; JAMA 320 (2018) 880-891. DOI: 10.1001/jama.2018.11473

29. Kamens, H.M., C.P. Silva, R.T. Nye, C.N. Miller, N. Singh, J. Sipko, N. Trushin, D. Sun, S.A. Branstetter, J.E. Muscat, J.P. Richie, and J. Foulds: Pharmacokinetic Profile of Spectrum Reduced Nicotine Cigarettes; Nicotine Tob. Res. (2019) ntz045.

DOI: $10.1093 / \mathrm{ntr} / \mathrm{ntz} 045$. [Epub ahead of print]

30. Benowitz, N.L., N. Nardone, K.M. Dains, S.M. Hall, S. Stewart, D. Dempsey, and P. Jacob: Effect of Reducing the Nicotine Content of Cigarettes on Cigarette Smoking Behavior and Tobacco Smoke Toxicant Exposure: 2-Year Follow Up; Addiction 110 (2015) 1667-1675. DOI: 10.1111/add.12978

31. Nardone, N., N.L. Benowitz, T.T. Smith, R. DenlingerApte, D.K. Hatsukami, J.S. Koopmeiners, Y. Ren, and E.C. Donny: Reasons for Non-Compliance in a Trial of Reduced Nicotine Cigarettes; Tob. Reg. Sci. 5 (2019) 87-93. DOI: 10.18001/TRS.5.1.8

32. Nardone, N., E.C. Donny, D.K. Hatsukami, J.S. Koopmeiners, S.E. Murphy, A.A. Strasser, J.W. Tidey, R. Vandrey, and N.L. Benowitz: Estimations and Predictors of Non-Compliance in Switchers to Reduced Nicotine Content Cigarettes; Addiction 111 (2016) 2208-2216. DOI: 10.1111/add.13519

33. Animal and Plant Health Inspection Service, USDA: Vector Tobacco; Availability of Determination of NonRegulated Status for Tobacco Genetically Engineered for Reduced Nicotine. 67 Fed. Reg. 71929, 2002. Available at: https:/www.federalregister.gov/docu ments/2002/02/12/02-3342/vector-tobacco-availabilityof-petition-and-environmental-assessment-for-determination-of (accessed July 25, 2019).

\section{Corresponding author:}

Ed Carmines, Ph.D.

Carmines Consulting, LLC,

Scottsdale, AZ, USA

E-mail: ed@carminesconsulting.com 